\title{
Ways to Develop Human-Level Web Intelligence: A Brain Informatics Perspective
}

\author{
Ning Zhong \\ Department of Life Science and Informatics \\ Maebashi Institute of Technology, Japan \& \\ The International WIC Institute/BJUT, China \\ zhong@maebashi-it.ac.jp
}

\begin{abstract}
In this paper, we briefly investigate several ways to develop human-level Web intelligence (WI) from a brain informatics (BI) perspective. BI can be regarded as brain sciences in WI centric IT age and emphasizes on a systematic approach for investigating human information processing mechanism. The recently designed instrumentation (fMRI etc.) and advanced IT are causing an impending revolution in both WI and BI, making it possible for us to understand intelligence in depth and develop human-level Web intelligence.
\end{abstract}

\section{Introduction}

The concept of Web intelligence (WI) was first introduced in our papers and books [13, 24, 27, 29, 31. Broadly speaking, WI is a new direction for scientific research and development that explores the fundamental roles as well as practical impacts of artificial intelligence (AI 1 and advanced information technology (IT) on the next generation of Web-empowered systems, services, and environments. The WI technologies revolutionize the way in which information is gathered, stored, processed, presented, shared, and used by virtualization, globalization, standardization, personalization, and portals.

As more detailed blueprints and issues of WI are being evolved and specified 13, 29, 31, 36, it has been recognized that one of the fundamental goals of WI research is to understand intelligence in depth and develop wisdom Web based intelligent systems that integrate all the human-level capabilities such as real-time response, robustness, autonomous interaction with their environment, communication with natural language, commonsense reasoning, planning, problem solving, decision making, learning, discovery and creativity.

Turing gave the first scientific discussion of human-level machine intelligence 23. Newell and Simon made a start on programming computers for general intelligence and investigated human problem solving in a behavior based approach [16. McCarthy argued that reaching human-level AI requires programs that deal with the commonsense informative situation, in which the phenomena

\footnotetext{
${ }^{1}$ Here the term of AI includes classical AI, computational intelligence, and soft computing etc.
} 
to be taken into account in achieving a goal are not fixed in advance [15. Laird and Lent proposed using interactive computer games that are the killer application for human-level AI research, because they can provide the environments for research on the right kinds of problem that lead to the type of incremental and integrative research needed to achieve human-level AI [10.

The new generation of WI research and development needs to understand multiple natures of intelligence in depth, by studying integrately the three intelligence related research areas: machine intelligence, human intelligence, and social intelligence, as shown in Figure 1 towards developing truly human-level Web intelligence. Machine intelligence (also called AI) has been mainly studied as computer based technologies for the development of intelligent knowledge based systems; Human intelligence (also called brain sciences) studies the nature of intelligence towards our understanding of intelligence; Social intelligence needs a combination of machine intelligence and human intelligence for establishing social networks that contain communities of people, organizations, or other social entities [29]. Furthermore, the Web can be regarded as a social network in which the Web connects a set of people (or organizations or other social entities). People are connected by a set of social relationships, such as friendship, co-working or information exchange with common interests. In other words, it is a Web-supported social network or called virtual community. In this sense, the study of WI is of social network intelligence (social intelligence for short).

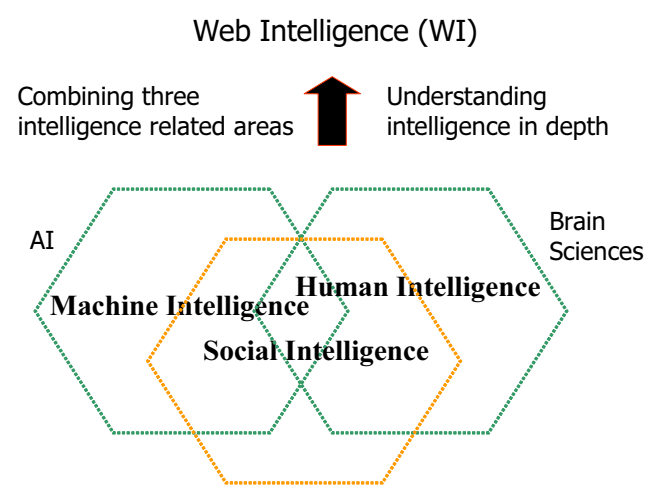

Fig. 1. The relationship between WI and other three intelligence related research areas

In the rest of the paper, we briefly investigate three ways to develop humanlevel Web intelligence from a brain informatics (BI) perspective. BI can be regarded as brain sciences in WI centric IT age [34,35. Although brain sciences have been studied from different disciplines such as cognitive science and neuroscience, BI represents a potentially revolutionary shift in the way that research is undertaken. BI is proposing to study human brain from the viewpoint of informatics (i.e. human brain is an information processing system) and use informatics (i.e. WI centric information technology) to support brain science study, in particular, WI provides urgent research needs. 


\section{Web Based Problem Solving with Human Level Capabilities}

A more concrete issue of WI is the development and application of a Webbased problem-solving system for portal-centralized, adaptable Web services [8, 13, 22, 29, 31.

Problem-solving is one of main capabilities of human intelligence and has been studied in both cognitive science and AI 16, where it is addressed in conjunction with reasoning centric cognitive functions such as attention, control, memory, language, reasoning, learning, and so on, using a logic based symbolic and/or connectionist approach. Although logic based problem-solving is "perfect", mathematical systems with no real time and memory constraints, Webbased problem-solving systems need real-time and dealing with global, multiple, huge, distributed information sources.

The core of such a system rests on the Problem Solver Markup Language (PSML) and PSML-based distributed Web inference engines for network reasoning, in which the following essential support functions should be provided:

- The expressive power and functional support in PSML for complex adaptive, distributed problem solving;

- Performing automatic reasoning on the Web by incorporating globally distributed contents and meta-knowledge, automatically collected and transformed from the semantic Web and social networks, with locally operational knowledge-data bases;

- Representing and organizing multiple, large-scale knowledge-data sources for distributed network reasoning;

- Combining multiple reasoning methods in PSML representation and distributed inference engines, efficiently and effectively;

- Modeling user behavior and representing/managing it as a personalized model dynamically;

- Including an emotional factor in developing the Web based reasoning and problem solving system.

A possible way as an immediate step to implement certain distributed reasoning capabilities of the future PSML is to make use of an existing logic language coupled with agent technologies. We have demonstrated one possible implementation of such capabilities. In particular, our proposed implementation, called $\beta$-PSML, is based on the combination of OWL with Horn clauses, and able to couple global semantic Web/social networks with local information sources for solving problems in a large-scale distributed Web environment [21,22].

Furthermore, in order to develop a Web based problem-solving system with human level capabilities, we need to better understand how human being does complex adaptive, distributed problem solving and reasoning, as well as how intelligence evolves for individuals and societies, over time and place [20,26, 35. In other words, ignoring what goes on in human brain and focusing instead on behavior has been a large impediment to understand how human being does complex adaptive, distributed problem solving and reasoning. 
In the light of BI, we need to investigate specifically the following issues:

- What are the existing thinking/reasoning models in AI, cognitive science, and neuroscience?

- How to design fMRI/EEG experiments and analyze such fMRI/EEG data to understand the principle of human reasoning and problem solving in depth?

- How to build the cognitive model to understand and predict user profile and behavior?

- How to implement human-level reasoning and problem solving on the Web based portals that can serve users wisely?

As a result, the relationships between classical problem solving and reasoning and biologically plausible problem solving and reasoning need to be defined and/or elaborated.

\section{Reasoning Centric Thinking Oriented Studies in Human Information Processing System}

Human intelligence related research studies the nature of intelligence towards our understanding of intelligence. The capabilities of human intelligence can be broadly divided into two main aspects: perception and thinking. So far, the main disciplines with respect to human intelligence are cognitive science that mainly focuses on studying mind and behavior based cognitive models of intelligence, as well as neuroscience that mainly focuses on studying brain and biological models of intelligence. In cognitive neuroscience, although many advanced results with respect to "perception oriented" study have been obtained, only a few of preliminary, separated studies with respect to "thinking oriented" and/or a more whole information process have been reported [6]. Figure 2 gives a global picture on reasoning centric thinking oriented functions and their relationships in human information processing system.

Our purpose is to understand activities of human information processing system by investigations in the following two levels:

- investigating the spatiotemporal features and flow of human information processing system, based on functional relationships between activated areas of human brain for each given task;

- investigating neural structures and neurobiological processes related to the activated areas [19].

More specifically, at the current stage, we want to understand:

- how a peculiar part (one or more areas) of the brain operates in a specific time;

- how the operated part changes along with time;

- how the activated areas work cooperatively to implement a whole information processing; 


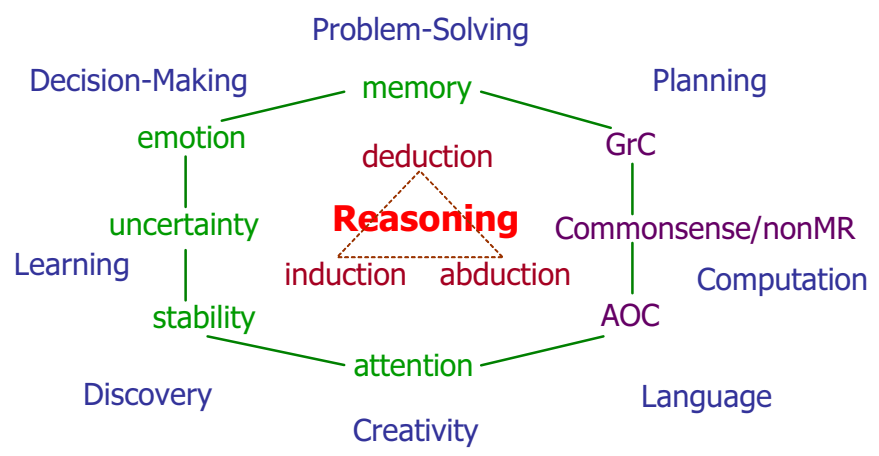

Fig. 2. Reasoning centric thinking oriented functions and their relationships ( $\mathrm{GrC}$ : Granular Computing 25]; AOC: Autonomy Oriented Computing [14]; nonMR: nonmonotonous reasoning)

- how the activated areas are linked, indexed, navigated functionally, and what are individual differences in performance;

- how a cognitive process is supported by neurobiological processes.

We need to study experimental cognitive neuroscience, data mining, intelligent agents, data and knowledge grids, the semantic Web and wisdom Web in a unified way 1, 2, 3, 4, 11, 12, 28, 34. We have been developing a full process from designing fMRI/EEG experiments based on WI needs for discovering new cognitive WI models. Such a full process means a systematic approach for measuring, collecting, modeling, transforming, managing, and mining multiple human brain data obtained from various cognitive experiments by using fMRI and EEG 33, 34.

As a step in this direction, we observe that fMRI brain imaging data and EEG brain wave data extracted from human information processing mechanism are peculiar ones with respect to a specific state or the related part of a stimulus. Based on this point of view, we propose a way of peculiarity oriented mining $(P O M)$ for knowledge discovery in multiple human brain data, without using conventional imaging processing to fMRI brain images and frequency analysis to EEG brain waves [17, 32, 33, 34. The proposed approach provides a new way for automatic analysis and understanding of fMRI brain images and EEG brain waves to replace human-expert centric visualization. The mining process is a multi-step one, in which various psychological experiments, physiological measurements, data cleaning, modeling, transforming, managing, and mining techniques are cooperatively employed to investigate human information processing mechanism.

Figure 3 gives the global picture of an example about how to investigate the spatiotemporal features and flow of human information processing system. In the cognitive process from perception to reasoning, data are collected in several event-related time points, and transformed into various forms in which POM 


\section{Thinking $\leftarrow-----\rightarrow$ Perception}

Reasoning - Language - Memory - Attention - Vision

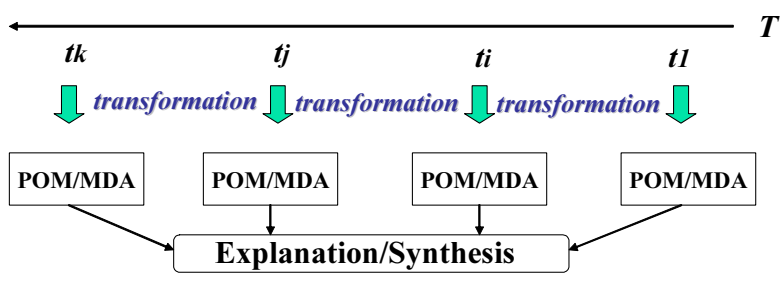

Fig. 3. Investigating the spatiotemporal features and flow of human information processing system

centric multi-aspect data analysis (MDA) can be carried out efficiently and effectively. Furthermore, the results of separate analysis can be explained and combined into a whole flow.

\section{A Data-Brain Model and Its Construction}

The Data-Brain is a brain database with all of data related to all major aspects and capabilities of human information processing mechanism for systematic investigation and understanding of human intelligence. The Data-Brain provides a holistic view at a long-term, global field of vision to understand the principle, models and mechanisms of human information processing system [9, 34, 35].

The key questions are how to obtain such data by systematic fMRI/EEG experiments, how to manage such huge multimedia data for systematic investigation and understanding of human intelligence, as well as how to analyse such data from multi-aspect and multi-level for discovering new cognitive models. A new conceptual model is needed to represent complex relationships among multiple human brain data sources, which are obtained by systematic fMRI/EEG experiments. Furthermore, the following supporting capabilities are requested to build such a Data Brain:

- It is a grid-based, simulation and analysis oriented, dynamic, spatial and multimedia database;

- It deals with multiple data sources, multiple data forms, multiple levels of data granulation;

- It provides multiple views and organizations;

- It includes various methods for data analysis, simulation, visualization, as well as corresponding knowledge and models.

At first, agents for data collecting, storing and retrieving are deployed on the Grid platform, like Globus, as a standard Grid service. OGSA-DAI is used to 
build database access applications [5 37. The aim of OGSA-DAI is to provide the middleware glue to interface existing databases, other data resources and tools to each other in a common way based on the Open Grid Services Architecture (OGSA). This middleware is based on the GGF-defined OGSI specification and layered on top of the Globus toolkit 3 OGSI implementation (GT3 Core).

Multiple data sources are collected by various cognitive fMRI/EEG experiments, modeling and transformation, and they are recorded to the corresponding databases through the Grid service on the distributed sites. Furthermore, the data-flow is a collection of descriptions for the dynamic relationship among multiple data sources on the data-grid. In the current study, data sources from cognitive fMRI/EEG experiments, to be collected on the data-grid, include:

- human multi-perception mechanism for studying the relevance between auditory and visual information processing;

- human deductive/inductive reasoning mechanism for understanding the principle of human reasoning and problem solving in depth;

- human computation mechanism as an example of human problem solving system;

- human decision-making mechanism from developing Web based decisionmaking support system with an emotional factor;

- human learning mechanism for acquiring personalized student models in an interactive learning process dynamically and naturally.

In order to build a Data Brain, a systematic methodology of cognitive experimental design needs to be developed, so that multiple human brain data sources obtained by fMRI/EEG experiments are interrelated and can be utilized for multi-purpose, not only a specific one. Event-related experimental designs have become an important methodology in EEG/fMRI research to evaluate the high level characteristics of human information processing in the central nervous system [18. There are, at present, two main methods called event-related potential (ERP) and event-related fMRI for event-related experimental designs. ERP is a tiny signal embedded in the ongoing EEG. By averaging the traces, investigators can extract this signal, which reflects neural activity that is specifically related cognitive events [7. ERPs are best suited for addressing questions about the time course of cognition rather than elucidating the brain structures that produce the electrical events. ERPs also provide physiological indices of when a person decides to response, or when an error is detected. On the other hand, event-related fMRI follows the same logic as used in ERP/EEG studies and provides the spatial resolution. Thus, event-related fMRI will further allow fMRI and EEG to be combined in paradigms that are identical across methods. By using such techniques, it is now becoming possible to study the precise spatiotemporal orchestration of neuronal activity associated with perceptual and cognitive events [18, as well as systematic collection of human brain data for building a Data Brain. 


\section{Conclusion}

BI emphasizes on a systematic approach for investigating human information processing mechanism, including measuring, collecting, modeling, transforming, managing, and mining multiple human brain data obtained from various cognitive experiments by using fMRI and EEG. The proposed methodology attempts to change the perspective of cognitive/brain scientists from a single type of experimental data analysis towards a holistic view at a long-term, global field of vision to understand the principle, models and mechanisms of human information processing. New generations of WI research and development need to understand multi-nature of intelligence in depth. The recently designed instrumentation (fMRI etc.) and advanced IT are causing an impending revolution in both WI and BI, making it possible for us to understand intelligence in depth and develop human-level Web intelligence.

\section{Acknowledgments}

I would like to express my gratitude to Enrico Franconi, Michael Kifer, Wolfgang May, Dieter Fensel and other organizers of ESWC 2007 for the kind invitation and the excellent organization. I am grateful to all my research collaborators, assistants, and students who have, over the years, together contributed to the development of Web Intelligence (WI) and Brain Informatics (BI). Special thanks to Jiming Liu, Yiyu Yao, Jinglong Wu, Benjamin Wah, and Shengfu Lu for our joint projects and discussions. I am very grateful to people who have joined or supported the WI and BI communities, members of the WIC advisory board, WIC technical committee, and WIC research centres, as well as keynote/invited speakers of IEEE/WIC/ACM WI-IAT conferences. This work is partially supported by the grant-in-aid for scientific research (No. 18300053) from the Japanese Ministry of Education, Culture, Sports, Science and Technology.

\section{References}

1. T. Berners-Lee, J. Hendler, O. Lassila, "The Semantic Web", Scientific American, 284, 34-43 (2001).

2. M. Cannataro and D. Talia, "The Knowledge Grid", Communications of the ACM, 46 (2003) 89-93.

3. D. Fensel, Ontologies: A Silver Bullet for Knowledge Management and Electronic Commerce, Springer (2001).

4. D. Fensel, "Unifying Reasoning and Search to Web Scale", IEEE Internet Computing, 11(2) (2007) 94-96.

5. I. Foster and C. Kesselman (eds.) The Grid: Blueprint for a New Computing Infrastructure, Morgan Kaufmann (1999).

6. M.S. Gazzaniga (ed.) The Cognitive Neurosciences III, The MIT Press (2004).

7. T.C. Handy, Event-Related Potentials, A Methods Handbook, The MIT Press (2004). 
8. J. Hu and N. Zhong, "Organizing Multiple Data Sources for Developing Intelligent e-Business Portals", Data Mining and Knowledge Discovery, Vol. 12, Nos. 2-3, Springer (2006) 127-150.

9. S.H. Koslow and S. Subramaniam (eds.) Databasing the Brain: From Data to Knowledge, Wiley (2005).

10. J.E. Laird and M. van Lent, "Human-Level AI's Killer Application Interactive Computer Games", AI Magazine (Summer 2001) 15-25.

11. Y. Li and N. Zhong, "Mining Ontology for Automatically Acquiring Web User Information Needs", IEEE Transactions on Knowledge and Data Engineering, Vol. 18, No. 4 (2006) 554-568.

12. J. Liu, N. Zhong, Y.Y. Yao, and Z.W. Ras, "The Wisdom Web: New Challenges for Web Intelligence (WI)", Journal of Intelligent Information Systems, 20(1) Kluwer (2003) 5-9.

13. J. Liu, "Web Intelligence (WI): What Makes Wisdom Web?", Proc. Eighteenth International Joint Conference on Artificial Intelligence (IJCAI'03) (2003) 15961601.

14. J. Liu, X. Jin, and K.C. Tsui, Autonomy Oriented Computing: From Problem Solving to Complex Systems Modeling, Springer (2005).

15. J. McCarthy, "Roads to Human Level AI?", Keynote Talk at Beijing University of Technology, Beijing, China (September 2004).

16. A. Newell and H.A. Simon, Human Problem Solving, Prentice-Hall (1972).

17. M. Ohshima, N. Zhong, Y.Y. Yao, and C. Liu, "Relational Peculiarity Oriented Mining", Data Mining and Knowledge Discovery, Springer (in press).

18. B.R. Rosen, R.L. Buckner, and A.M. Dale, "Event-related functional MRI: Past, Present, and Future", Proceedings of National Academy of Sciences, USA, Vol. 95, Issue 3 (1998) 773-780.

19. R.G. Shulman and D.L. Rothman, "Interpreting Functional Imaging Studies in Terms of Neurotransmitter Cycling", Proceedings of National Academy of Sciences, USA, Vol. 95, Issue 20 (1998) 11993-11998.

20. R.J. Sternberg, J. Lautrey, and T.I. Lubart, Models of Intelligence, American Psychological Association (2003).

21. Y. Su, L. Zheng, N. Zhong, C. Liu, and J. Liu, "Distributed Reasoning Based on Problem Solver Markup Language (PSML): A Demonstration through Extended OWL", Proc. 2005 IEEE International Conference on e-Technology, e-Commerce and e-Service (EEE'05), IEEE Press (2005) 208-213.

22. Y. Su, J. Liu, N. Zhong, L. Zheng, and C. Liu, "A Method of Distributed Problem Solving on the Web", Proc. 2005 IEEE/WIC/ACM International Conference on Web Intelligence (WI'05), IEEE Press (2005) 42-45.

23. A. Turing, "Computing Machinery and Intelligence", Mind LIX(236) (1950) 433-460.

24. Y.Y. Yao, N. Zhong, J. Liu, and S. Ohsuga, "Web Intelligence (WI): Research Challenges and Trends in the New Information Age", N. Zhong, Y.Y. Yao, J. Liu, S. Ohsuga (eds.) Web Intelligence: Research and Development, LNAI 2198, Springer (2001) 1-17.

25. Y.Y. Yao and N. Zhong, Granular Computing Using Information Tables. In T.Y. Lin, Y.Y. Yao, L.A. Zadeh (eds.) Data Mining, Rough Sets and Granular Computing, Physica-Verlag (2002) 102-124.

26. L.A. Zadeh, "Precisiated Natural Language (PNL)", AI Magazine, 25(3) (Fall 2004) 74-91. 
27. N. Zhong, J. Liu, Y.Y. Yao, and S. Ohsuga, "Web Intelligence (WI)", Proc. 24th IEEE Computer Society International Computer Software and Applications Conference (COMPSAC 2000), IEEE Press (2000) 469-470.

28. N. Zhong, C. Liu, and S. Ohsuga, "Dynamically Organizing KDD Process", International Journal of Pattern Recognition and Artificial Intelligence, Vol. 15, No. 3, World Scientific (2001) 451-473.

29. N. Zhong, J. Liu, and Y.Y. Yao, "In Search of the Wisdom Web", IEEE Computer, 35(11) (2002) 27-31.

30. N. Zhong, "Representation and Construction of Ontologies for Web Intelligence", International Journal of Foundations of Computer Science, World Scientific, Vol. 13, No. 4 (2002) 555-570.

31. N. Zhong, J. Liu, and Y.Y. Yao (eds.) Web Intelligence, Springer, 2003.

32. N. Zhong, Y.Y. Yao, and M. Ohshima, "Peculiarity Oriented Multi-Database Mining", IEEE Transaction on Knowlegde and Data Engineering, Vol. 15, No. 4 (2003) 952-960.

33. N. Zhong, J.L. Wu, A. Nakamaru, M. Ohshima, and H. Mizuhara, "Peculiarity Oriented fMRI Brain Data Analysis for Studying Human Multi-Perception Mechanism", Cognitive Systems Research, 5(3), Elsevier (2004) 241-256.

34. N. Zhong, J. Hu, S. Motomura, J.L. Wu, and C. Liu, "Building a Data Mining Grid for Multiple Human Brain Data Analysis", Computational Intelligence, 21(2), Blackwell Publishing (2005) 177-196.

35. N. Zhong, "Impending Brain Informatics (BI) Research from Web Intelligence (WI) Perspective", International Journal of Information Technology and Decision Making, World Scientific, Vol. 5, No. 4 (2006) 713-727.

36. N. Zhong, J. Liu, and Y.Y. Yao, "Envisioning Intelligent Information Technologies (iIT) from the Stand-Point of Web Intelligence (WI)", Communications of the ACM, 50(3) (2007) 89-94.

37. The OGSA-DAI project: http://www.ogsadai.org.uk/. 